$63^{\text {ème }}$ Congrès de la SFCO, 02025 (2015)

DOI:10.1051/sfco/20156302025

(C) Owned by the authors, published by EDP Sciences, 2015

\title{
COMMUNICATION
}

\section{Hémangiome intraosseux mandibulaire : à propos d'un cas}

\section{Soto F, Dekeister C}

CHU Toulouse, Hôpital Pierre Paul Riquet, Service de chirurgie maxillo faciale, Place du Dr Baylac TSA 40031, 31059 Toulouse

Les hémangiomes intraosseux maxillo-mandibulaires sont des tumeurs bénignes rares, à haut risque hémorragique lors de leurs explorations diagnostiques.

La physiopathologie de l'hémangiome s'explique par une perturbation vasculaire précoce pendant l’embryogénèse.

Il faut le différencier des malformations artérioveineuses (MAV), secondaires, quant à elles, à une perturbation tardiveau cours de l'embryogenèse, avec persistance d'anastomoses artérioveineuses.

L'hémangiome présente une croissance rapide dans l'enfance et une phase de stabilisation à l'âge adulte. Il peut spontanément régresser, à l'inverse des MAV.

Les auteurs présentent le cas d'une patiente de 56 ans consultant pour une légère déformation mandibulaire, découverte il y a deux mois par autopalpation.

Il s'agit d'une tuméfaction du rebord basilaire gauche, sans autre symptôme associé.

L'examen clinique exofacial ne retrouve pas de répercussion esthétique du tiers inferieur de la face. La lésion est palpable en endobuccal, en regard des apex de la canine à la deuxième prémolaire du secteur 3. Les dents concernées ont un test de vitalité positif et sont non mobiles.

L'orthopanthomogramme et le cone-beam retrouvent une image bien délimitée en rayon de miel, de $2 \mathrm{~cm}$ de grand axe, avec une dédifférenciation cortico-médullaire au niveau du rebord basilaire.

La lésion est à distance du nerf alvéolaire inférieur et des apex dentaires.

L'angioIRM dynamique retrouve une vascularisation intra-lésionnelle peu intense, sans extension péri-nerveuse.

Après discussion pluridisciplinaire, devant le caractère potentiellement évolutif et le risque hémorragique modéré, une biopsie sous anesthésie générale est réalisée.

L'analyse anatomopathologique retrouve la présence de structures vasculaires capillaires caractéristique d'un hémangiome caverneux.

Il s'agit donc ici d'une lésion asymptomatique, en dehors d'une discrète déformation osseuse, à faible risque neurologique, fracturaire ou hémorragique.

De plus, connaissant l'histoire naturelle de cette lésion, souvent stable et pouvant régresser, une surveillance radio-clinique est décidée.

Actuellement, il n'existe pas de consensus concernant la prise en charge de l'hémangiome mandibulaire.

Celle-ci dépend de différentes modalités, comme l’âge du patient, le risque fracturaire, le degré de déformation, et ses rapports aux structures dentaires et nerveuses.

La procédure la plus fréquemment pratiquée est l'embolisation sélective, suivie d’une résection chirurgicale en bloc. Néanmoins, cette intervention peut-être mutilante.

This is an Open Access article distributed under the terms of the Creative Commons Attribution License 4.0, which permits unrestricted use, distribution, and reproduction in any medium, provided the original work is properly cited. 
Dans la littérature d'autres alternatives médico-chirurgicales sont proposées, comme la résection partielle, la cryothérapie ou l'injection d'agent sclérosant.

Certains de ces protocoles nécessitent des plateaux techniques spécifiques.

Cependant, une surveillance radio-clinique peut aussi s'envisager pour certains hémangiomes mandibulaires, peu symptomatiques et à faible risque de complication à moyen terme.

En fonction de l'évolutivité de la lésion, les thérapeutiques usuelles peuvent secondairement être envisagées.

La prise en charge de l'hémangiome caverneux reste donc une décision multidisciplinaire prenant en compte les caractéristiques de la lésion et le plateau technique disponible.

Références

- $\quad$ Larsen et Peterson (1993)

- Williams et al. (2002)

- Eliot et Castle (2010)

- Cheng et al. (2006)

- Oliveira et al. (2008)

- Noreau et al. (2001)

- Wu et al. (2011)

- Kalsi et Scannell (2013)

Nom et adresse du conférencier

Florie SOTO

CHU Toulouse, Hôpital Pierre Paul Riquet, Service de chirurgie maxillo faciale

Place du Dr Baylac TSA 40031

31059 Toulouse (France)

floriesoto@hotmail.fr 